\title{
Erratum: Breakdown of Hund's third rule in amorphous Co-W nanoparticles and crystalline $\mathrm{Co}_{3} \mathrm{~W}$ alloys [Phys. Rev. B 86, 064428 (2012)]
}

\author{
A. I. Figueroa, F. Bartolomé, J. Bartolomé, L. M. García, F. Petroff, C. Deranlot, F. Wilhelm, and A. Rogalev \\ (Received 12 April 2013; published 26 April 2013)
}

DOI: 10.1103/PhysRevB.87.139906

PACS number(s): 75.75.Fk, 75.50.Kj, 71.20.Be, 78.70.Dm, 99.10.Cd

In our manuscript we describe the strong relationship between the structural properties of a material and the breakdown of Hund's third rule, as found for amorphous and crystalline Co-W alloy systems. We compare our findings with the work of Kapaklis et al. ${ }^{1}$ in amorphous $\mathrm{Co}(\mathrm{Fe})-\mathrm{Zr}$ and crystalline $\mathrm{CoZrPt}$ alloys, where similar effects are observed. However, the interpretation and comparison of the conclusions of the latter work are not properly addressed in our manuscript. In fact, even though the work by Kapaklis et al. ${ }^{1}$ shows differences for the amorphous and crystalline cases, while our work describes analogous behavior for both kinds of samples, these two studies lead to the same conclusion: The breakdown of Hund's third rule is not caused by the crystallinity or disorder level of the material, but, more specifically, it results from the reduction of the interatomic distance between the magnetic and nonmagnetic atoms. Therefore, both papers contribute significantly to evidence the influence of the structure and chemical environment on the induced orbital moments.

As a result of the aforementioned reasons, the statement in our paper, "In the same study, Kapaklis et al. claim that the crystallinity of the material is determinant in the breakdown of Hund's third rule since $\mathrm{Zr}$ induced moments in crystalline CoZrPt alloys are found to fulfill the rule," should be disregarded.

${ }^{1}$ V. Kapaklis, P. T. Korelis, B. Hjörvarsson, A. Vlachos, I. Galanakis, P. Poulopoulos, K. Özdoğan, M. Angelakeris, F. Wilhelm, and A. Rogalev, Phys. Rev. B 84, 024411 (2011). 